\title{
ARC syndrome
}

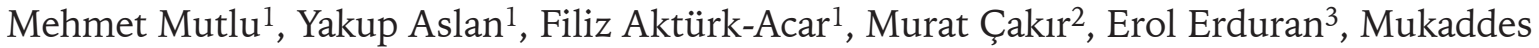
Kalyoncu $^{4}$

Divisions of ${ }^{1}$ Neonatology, ${ }^{2}$ Pediatric Gastroenterology Hepatology and Nutrition, ${ }^{3}$ Pediatric Hematology and Oncology, ${ }^{4}$ Pediatric Nephrology and Rheumatology, Karadeniz Technical University, Faculty of Medicine, Trabzon, Turkey.

E-mail: drmehmetmutlu38@hotmail.com

Received: 12th January 2017, Revised: 7th February 2017, Accepted: 7th February 2017

SUMMARY: Mutlu M, Aslan Y, Aktürk-Acar F, Çakır M, Erduran E, Kalyoncu M. ARC syndrome. Turk J Pediatr 2017; 59: 487-490.

Arthrogryposis-renal dysfunction-cholestasis (ARC) is an autosomal recessive multisystem disorder characterized by arthrogryposis, renal tubular dysfunction and neonatal cholestasis with low gamma glutamyl transpeptidase activity. Most of the mutations in ARC syndrome are associated with the vacuolar protein sorting 33B (VPS33B) gene on chromosome 15q26.1. Herein, we report a female newborn with ARC syndrome caused by homozygous mutations in VPS33B [IVS1-2A >C (c.97-2A >C)].

Key words: arthrogryposis, cholestasis, neonate, renal dysfunction.

Arthrogryposis-renal dysfunction-cholestasis (ARC) syndrome is a rare, fatal, multisystem disorder that mainly effects the liver, kidney, skin, central nervous and musculoskeletal systems. This progressive disorder has autosomal recessive inheritance and is commonly caused by a mutation in the vacuolar protein sorting 33B (VPS33B) gene on chromosome $15 \mathrm{q} 26.1{ }^{1}$ This syndrome is also caused by a mutation in VIPAR (also called C14ORF133) in individuals with ARC syndrome without VPS33B defects. ${ }^{2}$ Additional clinical features of the disease are ichthyosis, platelet abnormality, agenesis of the corpus callosum, congenital cardiovascular anomalies, deafness, recurrent sepsis, hypothyroidism and nephrogenic diabetes insipidus. ${ }^{3}$

Herein, we report a female newborn with ARC syndrome caused by a homozygous mutation in VPS33B [IVS1-2A >C (c.97-2A >C)] gene.

\section{Case Report}

A 14-day-old female neonate was admitted to the neonatal intensive care unit with jaundice. She was born at $40^{\text {th }}$ week of gestation via caesarean section with a birth weight of 3,360 g. Her parents were cousin. On physical examination; dysmorphic features [low set ear, high arched palate, beaked nose, micrognathia, rigid kyphosis (Fig. 1a), radial deviation of the wrist (Fig. 1b), flexion contracture of the left elbow, talipes calcaneovalgus (Fig. 1c)], heart murmur, jaundice, dry and scaly skin like ichthyosis, and hepatomegaly were noted. On laboratory examination, hemoglobin was $12.2 \mathrm{~g} / \mathrm{dl}$, leucocytes counts $11,000 / \mathrm{mm}^{3}$ and platelet counts $331,000 / \mathrm{mm}^{3}$. Review of a peripheral blood smear revealed large and pale platelets (Fig. 2). Activated partial thromboplastin time, prothrombin time and international normalized ratio were 25.6 secs (23.6 - 34.8), 13 secs (11 - 15.5) and 1.04 (0.8 - 1.25), respectively. Bleeding time was 14 minutes. Platelet aggregation showed abnormal responses to epinephrine and adenosine diphosphate (ADP) but normal responses to collagen and ristocetin.

Biochemical analyses showed: glucose 102 $\mathrm{mg} / \mathrm{dl}(74$ - 106), blood urea nitrogen 11 $\mathrm{mg} / \mathrm{dl}(6-20)$, creatinine $0.64 \mathrm{mg} / \mathrm{dl}(0.51$ - 0.95), eGFR $35 \mathrm{ml} / \mathrm{dk} / 1.73 \mathrm{~m}^{2}$, alaninine aminotransferase (ALT) $19 \mathrm{U} / \mathrm{L}(0-45)$, aspartate aminotransferase (AST) $32 \mathrm{U} / \mathrm{L}(0$ - 35), total /direct bilirubin 11.24/5.92 mg/ dl $(0.3-1.2) /(0-0.2)$, alkaline phosphatase $674 \mathrm{U} / \mathrm{L}(48$ - 406), $\gamma$-glutamyl transpeptidase $(\gamma \mathrm{GT}) 26 \mathrm{U} / \mathrm{L}(0-55)$, total protein $5.3 \mathrm{~g} /$ dl (6.6 - 8.3), albumin $3.4 \mathrm{~g} / \mathrm{dl}(3.5$ - 5.2), 
phosphate $3.64 \mathrm{mg} / \mathrm{dl}(2.5$ - 4.5), calcium 10.1 $\mathrm{mg} / \mathrm{dl}$ (8.8 - 10.6), creatine kinase $157 \mathrm{U} / \mathrm{L}$ (20-200), ferritin $651.3 \mathrm{ng} / \mathrm{ml}(11-306.8)$, and procalcitonin $0.44 \mu \mathrm{g} / \mathrm{L}(<0.5)$.

Thyroid function tests revealed that thyroidstimulating hormone $20.41 \mu \mathrm{IU} / \mathrm{ml}(0.27$ 4.2) and free thyroxine $1.68 \mathrm{ng} / \mathrm{dl}(0.93$ - 1.7). Plasma alpha 1 antitrypsin, alpha fetoprotein, sweat chloride concentration, amino acid analysis of blood, and tandem mass spectroscopy were normal.

Arterial blood gas analysis showed $\mathrm{pH} 7.34$, $\mathrm{pCO}_{2} 32.8 \mathrm{mmHg}, \mathrm{HCO}_{3} 18.8 \mathrm{mmol} / \mathrm{L}$, base excess $-7.2 \mathrm{mmol} / \mathrm{L}$.

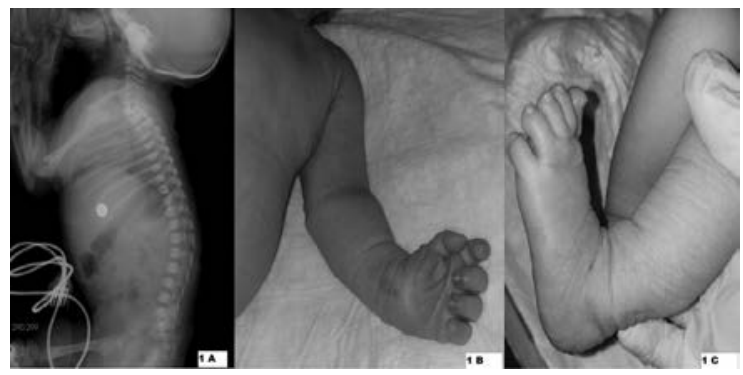

Fig. 1a. Rigid kyphosis 1b. Radial deviation of the wrist 1c. Talipes calcaneovalgus of the patient with ARC syndrome

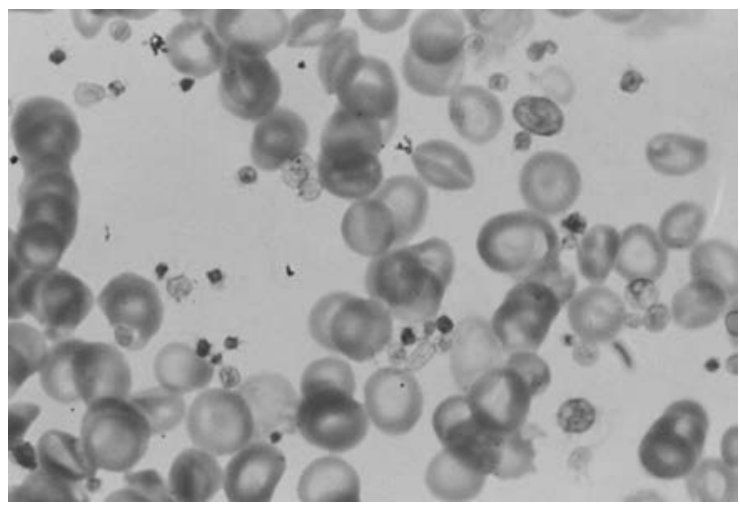

Fig. 2. Abnormally large homogenous, nongranular greyappearing platelets of the patient with ARC syndrome

Intron 1

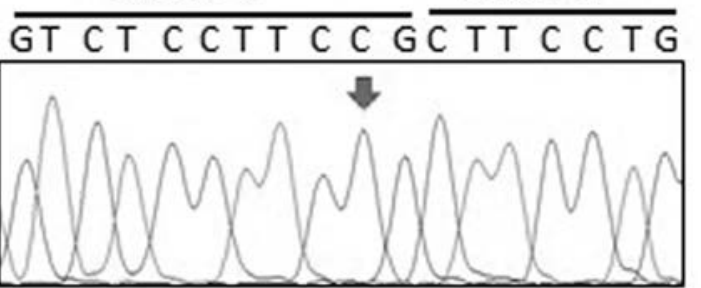

Fig. 3. Sequence chromatogram showing IVS1-2A $>C$ (c.97$2 \mathrm{~A}>\mathrm{C}$ ) splicing acceptor site mutation in VPS33B gene. Arrow shows the position of the substitution.
Urine and urine amino acid analysis showed glucosuria, generalized aminoaciduria, tubular proteinuria and phosphaturia. Urine specific gravity was 1.006. Tubular phosphate reabsorption ratio was $52 \%$. She had polyuria $(9.8 \mathrm{ml} / \mathrm{kg} / \mathrm{h})$.

Microbiologic evaluation for the antibodies against toxoplasmosis, rubella and cytomegalovirus was negative.

Echocardiography showed ventricular septal defect (VSD) and atrial septal defect (ASD). Ultrasonographic evaluations revealed dislocation of both hip joints and medullary nephrocalcinosis. Cranial magnetic resonance imaging was normal. Brainstem evoked response audiometry test was bilaterally negative.

Liver biopsy could not be performed due to the risk of bleeding. The diagnosis was confirmed by genetic analysis and the result was a homozygous mutation in VPS33B gene $($ NG_012162.1) [IVS1-2A $>$ C $($ c.97-2A $>$ C)] (Fig. 3).

The patient experienced three clinical episodes of sepsis in the hospitalization period. Despite appropriate antimicrobial therapy, the patient died at sixty days of her life due to a third sepsis episode. Klebsiella pneumoniae grew in the urine culture but not in the blood cultures in this sepsis episode. Genetic counseling was given to the patient's parents. A written consent form was obtained from the parents of the patient to present this case.

\section{Discussion}

In this manuscript; we report a neonate with a homozygous mutation in the VPS33B gene [IVS1$2 \mathrm{~A}>\mathrm{C}(\mathrm{c} .97-2 \mathrm{~A}>\mathrm{C})]$, with the classical clinical features of ARC with additional clinical features including ichthyosis, platelet abnormality, congenital cardiovascular anomalies, deafness, recurrent sepsis, hypotonia, hypothyroidism and failure to thrive. It is known that this mutation is defined in a patient that has unknown ethnic group. ${ }^{4}$ To our knowledge this case, with c.97$2 \mathrm{~A}>\mathrm{C}$ mutation, is the first reported patient with ARC in Turkey.

The genetic lesion responsible for the phenotype has been mapped; a germline mutation in the VPS33B gene; which is a member of the Sec-1/Munch 18 family that involved in vesicular trafficking among cell compartments 
in processes such as synaptic transmission, vesicular exocytosis, and general secretion. They modulate membrane fusion events by their strong interaction with the members of the syntaxin family. These proteins are widely expressed in throughout body including liver, skin, brain, kidneys and skeletal muscle which may explain the systemic symptoms observed in ARC syndrome. ${ }^{1}$

Arthrogryposis is a cardinal feature of ARC syndrome and manifests as radial deviation of the wrist, dislocation of the joint, vertical talus, flatfoot, pathologic fracture, rigid kyphosis, pes calcaneovalgus, muscular atrophy, and flexion contractures of the limbs. ${ }^{5}$ In our case; rigid kyphosis, radial deviation of the wrist, flexion contracture of the left elbow, dislocation of the hip joint and talipes calcaneovalgus were present.

Renal tubular dysfunction is another cardinal feature of ARC syndrome. It is characterized by renal tubular acidosis, nephrogenic diabetes insipidus, glucosuria, aminoaciduria and phosphaturia. ${ }^{3}$ Nephrocalcinosis may be seen on ultrasonograpic examination ${ }^{3}$. In our case, glucosuria, aminoaciduria, proteinuria, phosphaturia, polyuria, mild metabolic acidosis and nephrocalcinosis were present.

Neonatal cholestatic jaundice is another cardinal feature of ARC syndrome. Hepatomegaly, cholestatic jaundice with consistently low $\gamma \mathrm{GT}$ is the most common characteristic of ARC syndrome. ${ }^{3}$ However, ARC syndrome with high $\gamma$ GT levels caused by VPS33B mutation has been reported. ${ }^{6}$ AST and ALT levels may be normal or slightly elevated ${ }^{3}$. In our case, hepatomegaly, cholestatic jaundice with normal $\gamma \mathrm{GT}$, normal AST and ALT levels were present.

Life-threatening hemorrhage due to platelet dysfunction is an important clinical feature. Platelet function abnormalities are likely to be secondary to marked reduction of $\alpha$-granules biosynthesis. ${ }^{7}$ Platelet morphology is abnormal in ARC syndrome with large, agranular, and pale-appearing platelets seen in grey platelet syndrome. ${ }^{8}$ Our case had large, agranular, pale-appearing platelets on review of peripheral blood smear and had abnormal responses to epinephrine and ADP, but normal responses to collagen and ristocetin in platelet aggregation studies, as Saadah et al. reported. ${ }^{8}$
Organ biopsy can be dangerous because of the risk of severe bleeding in an ARC suspected patient. Mutation analysis should be preferred for diagnosis. ${ }^{9,10}$ Therefore, biopsy was not performed on the patient and the diagnosis was confirmed by genetic analysis.

Congenital heart disease and central nervous system anomalies can occasionally be seen in patients with ARC syndrome. ${ }^{3}$ Our patient had VSD and ASD, but had not central nervous system anomalies. The other additional features of ARC syndrome including ichthyosis, deafness, recurrent sepsis, and hypothyroidism were also present in our patient.

Unfortunately, no curative treatment of ARC syndrome is yet available. Current therapeutic strategies are supportive care including fluid infusion, antimicrobial treatment, fat-soluble vitamins and ursodeoxycholic acid, calcium glubionate, L-thyroxine and phosphate supplementation. ${ }^{3}$ Death occurs in the first year of life in most cases. These treatments were administered to our patient. Despite appropriate antimicrobial therapy, the patient died at sixty days of her life due to a third sepsis episode.

In summary; ARC syndrome should be kept in mind in patients with cholestatic jaundice with consistently low $\gamma \mathrm{GT}$, and musculoskeletal abnormality. Despite intensive management, clinical course in ARC syndrome is poor. Confirmation of diagnosis with genetic tests and genetic counseling to the affected families are important.

\section{REFERENCES}

1. Gissen P, Johnson CA, Morgan NV, et al. Mutations in VPS33B, encoding a regulator of SNARE-dependent membrane fusion, cause arthrogryposis-renal dysfunction-cholestasis (ARC) syndrome. Nat Genet 2004; 36: 400-404.

2. Cullinane AR, Straatman-Iwanowska A, Zaucker A, et al. Mutations in VIPAR cause an arthrogryposis, renal dysfunction and cholestasis syndrome phenotype with defects in epithelial polarization. Nat Genet 2010; 42: 303-312.

3. Zhou Y, Zhang J. Arthrogryposis-renal dysfunctioncholestasis (ARC) syndrome: From molecular genetics to clinical features. Ital J Pediatr 2014; 40: 77.

4. Smith H, Galmes R, Gogolina E, et al. Associations among genotype, clinical phenotype, and intracellular localization of trafficking proteins in ARC syndrome. Hum Mutat 2012; 33: 1656-1664. 
5. Jang WY, Cho TJ, Bae JY, et al. Orthopaedic manifestations of arthrogryposis-renal dysfunctioncholestasis syndrome. J Pediatr Orthop 2011; 31: 107-112.

6. Wang JS, Zhao J, Li LT. ARC syndrome with high GGT cholestasis caused by VPS33B mutations. World J Gastroenterol. 2014; 20: 4830-4834.

7. Lo B, Li L, Gissen P, et al. Requirement of VPS33B, a member of the Sec1/Munc18 protein family in megakaryocyte and platelet alpha-granule biogenesis. Blood 2005; 106: 4159-4166.
8. Saadah OI, Bokhari BE, Alshaeri TM, Jastaniah W. Haematological manifestations of arthrogryposis - renal dysfunction cholestasis (ARC) syndrome: A case report. Arab J Gastroenterol 2013; 14: 26-28.

9. Kim SM, Chang HK, Song JW, Koh H, Han SJ; Severance Pediatric Liver Disease Research Group. Agranular platelets as a cardinal feature of ARC syndrome. J Pediatr Hematol Oncol 2010; 32: 253-258.

10. Hayes JA, Kahr WH, Lo B, Macpherson BA. Liver biopsy complicated by hemorrhage in a patient with ARC syndrome. Paediatr Anaesth 2004; 14: 960-963. 